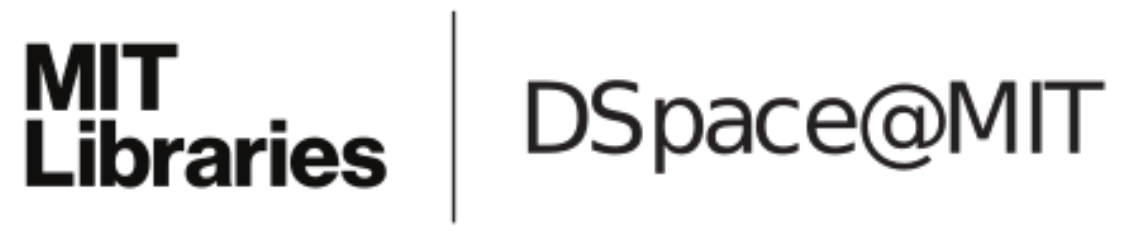

MIT Open Access Articles

Do we see more than we can access?

The MIT Faculty has made this article openly available. Please share how this access benefits you. Your story matters.

Citation: Alex Byrne, David R. Hilbert and Susanna Siegel (2007). Do we see more than we can access?. Behavioral and Brain Sciences, 30 , pp 501-502 doi:10.1017/S0140525X07002816

As Published: http://dx.doi.org/10.1017/S0140525X07002816

Publisher: Cambridge University Press

Persistent URL: http://hdl.handle.net/1721.1/50989

Version: Final published version: final published article, as it appeared in a journal, conference proceedings, or other formally published context

Terms of Use: Article is made available in accordance with the publisher's policy and may be subject to US copyright law. Please refer to the publisher's site for terms of use. 
phenomenal memory system, is greater than that of the working memory buffer that governs reporting" (sect. 9, para. 11), and (e) "the machinery of phenomenology is at least somewhat different from the machinery of cognitive accessibility" (sect. 9, para. 13). (That is, (a)-(b) entails (d)-(e), but not vice-versa.) Second, he argues for accepting Sperling-type experiments at face value. Third, he invokes neurological conjecture to support (c).

One might accept (a)-(b) and (d)-(e), but insist that working memory and "reportability" are constitutive to phenomenal consciousness. One might hold that although some specific phenomenally conscious items do not appear in working memory, all phenomenal consciousness depends constitutively on some items' being accessible to working memory. Block marshals neurological considerations for (c) against such a position.

In his second stage, Block opposes Dehaene's attempts to avoid taking Sperling-type experiments at face value. Block effectively criticizes postulating what he calls a refrigerator-light illusion, and points out that it is question-begging to invoke "change-blindness" to support the position that the subjects in Sperling-type experiments are under an illusion that they had phenomenal experiences of items that do not appear in working memory. The two cases are disanalogous in a way that Block does not note. On Dehaene's view that Sperling-type subjects are phenomenally conscious only of items actually in working memory, the subjects cannot have had, before the cue that selects those items retained in working memory, a phenomenologically conscious perception of any of the specific 8-32 items that they seem to have experienced. On that view, subjects' sense of having consciously perceived even specific retained items before they appear in working memory is illusion. No one postulates analogous total illusion in "change-blindness" cases. Even proponents of the (I think mistaken) view that items that change unnoticed are not consciously seen do not claim that nothing is consciously seen.

I believe that Sperling-type experiments support (c), not just (a), (b), (d), (e). I argue by dilemma. If retained and unretained items are held not to be conscious before any items are retained in working memory, what is the evidence that memory of their having been conscious is total illusion? Exposure is long enough for perceptual processing to be complete. Why should phenomenology, even of specific retained items, have been missing? We have independent evidence about working memory. It does have constructive functions: making consciousness more vivid, rehearsing to facilitate retention and reproduction of imagery (Andrade 2001; Pearson 2001). But its primary function is to preserve perception already formed. Holding that its preservations convey systematic illusion is ad hoc. The fact that subjects seem to remember having seen all items, and could be cued to retain any item specifically, supports believing that even specific unretained items are phenomenally conscious. Now suppose that all, or at least the retained, specific items are held to be conscious before being preserved in working memory. What is the evidence that mere accessibility to working memory is constitutive to their being occurrently conscious beforehand? Such a view labors under heavy empirical burden. Consciousness is an occurrent, not a dispositional, condition. We have no good idea how mere dispositional accessibility to working memory could be causally necessary to occurrence of consciousness before working memory operates. Why should the door's being open matter to the occurrence of something that does not use the door until after it already occurs? Such a view would require very special evidence and explanation. In the absence of specific empirical support, the idea is not a serious contender. The best explanation of current evidence is that conscious perception of the specifics of items later retained, indeed of all 8-32 items, occurs independently of working memory. The machinery of phenomenal consciousness appears to be independent of the machinery of working memory. Conclusion (c) is supported independently of Block's neurological conjecture.

Further evidence for (c) may lie in the formation speed of at least generic phenomenally conscious aspects of visual perception. Some super-ordinate object categorization occurs in less than $150 \mathrm{msec}$ - before a signal even reaches working memory (VanRullen \& Thorpe 2001; Rousselet et al. 2004a; 2004b). Such considerations are tentative. But it is important not to be so fixed on neurological matters that one underrates the force of psychological considerations in supporting psychological conclusions.

\section{Do we see more than we can access?}

\author{
doi: 10.1017/S0140525X07002816
}

Alex Byrne ${ }^{\mathrm{a}}$, David R. Hilbert ${ }^{\mathrm{b}}$, and Susanna Siegel
${ }^{\mathrm{a}}$ Department of Linguistics and Philosophy, Massachusetts Institute of
Technology, Cambridge, MA 02319; ${ }^{\mathrm{b}}$ Department of Philosophy, University of
Illinois at Chicago, MC 267, Chicago, IL 60607; ${ }^{\mathrm{C}}$ Department of Philosophy,
Harvard University, Emerson Hall, Cambridge, MA 02138.
abyrne@mit.edu http://web.mit.edu/abyrne/www
hilbert@uic.edu www.uic.edu/ hilbert
ssiegel@fas.harvard.edu http://www.people.fas.harvard.edu/ ssiegel

Abstract: One of Block's conclusions, motivated by partial-report superiority experiments, is that there is phenomenally conscious information that is not cognitively accessible. We argue that this conclusion is not supported by the data.

Block's overall argument appeals to the lemma that "in a certain sense phenomenal consciousness overflows cognitive accessibility" (target article, Abstract), which Block takes to be supported by Landman et al. (2003) and Sligte et al. (2008). (For reasons of space we will ignore the latter.) Block summarizes his discussion of these two papers as follows:

The main upshot of the Landman et al. and the Sligte et al. experiments (at least on the surface - debunking explanations will be considered later) is along the same lines as that of the Sperling experiment: The subject has persisting experiences as of more specific shapes than can be brought under the concepts required to report or compare those specific shapes with others. (sect. 9, para. 10)

Thus, in the first condition of the Landman et al. experiment, Block holds that the subjects have persisting experiences as of [a circle of] eight rectangles, with the horizontal/vertical orientation of each rectangle specified. And if that is so, then, as Block says, the subject's experiences are not completely accessible, because the subjects can report the orientation of only four (or so) rectangles.

Although most of Block's discussion is couched in terms of "phenomenal consciousness" and the like, for present purposes we can talk instead (as Block himself sometimes does) of what the subjects see. Put this way, Block's claim is that the subjects continue to see each rectangle as oriented horizontally or vertically after the stimulus has been replaced with a gray screen. In the terminology of Coltheart (1980), this is an example of visible persistence.

Coltheart distinguishes visible persistence from informational persistence. The latter is defined not in terms of seeing, or phenomenal consciousness, but in terms of the persistence of rich visual information about a stimulus after it has been replaced. Sperling-type experiments show that stimulus information is held in a high-capacity but transient memory, and thus that there is informational persistence. One might hold that there is informational persistence simply because there is visible persistence; that is, stimulus information continues to be available because the subject continues to see the stimulus. Coltheart argues, however, that the phenomena are not connected so intimately. One consideration is that informational persistence lasts longer than a few hundred ms, the duration of visible persistence. (As Block notes, the duration of informational persistence found by Landman et al. is about 1,500 msecs. $)^{1}$ 
Commentary/Block: Consciousness, accessibility, and the mesh

With this distinction in hand, consider Landman et al.'s conclusion:

The present data agree with the presence of two parallel types of short term memory. ... Almost all items enter the first type of memory. It is like iconic memory, because it has a high capacity and it is maskable.... The second type of memory is one that resists interference by new stimuli. When new items enter the visual system, they replace the old items, except the ones that have entered the second type of representation. ... The cue-advantage arises because the subjects selectively transfer the cued item from iconic memory to the more durable working memory. ... (Landman et al. 2003, p.162)

Landman et al. are, then, concerned with informational persistence, not visible persistence. Their paper contains no data concerning visible persistence. Since informational persistence is consistent with no visible persistence at all, Block's appeal to Landman et al. must be somewhat indirect.

And indeed it is. Block's argument for visible persistence is based on subjects' reports: "[subjects say they are] continuing to maintain a visual representation of the whole array" (sect. 9, para. 6).

We have three points about this. First, Block needs only the weaker claim that the subjects in the Landman et al. experiment saw each rectangle as oriented horizontally or vertically, not the stronger claim that the subjects remain in this state after the stimulus has been replaced. The weaker claim implies Block's conclusion about inaccessibility for the same reason that the stronger one does.

We do not dispute that information about the orientation of each rectangle persists and is not as a whole accessible; we do dispute Block's claim that this inaccessible information characterizes what the subjects see. Our second point is that it is unclear that subjects' reports unequivocally support Block. Block needs subjects to agree that they saw each rectangle as oriented horizontally or vertically (even if they can't report which orientation each rectangle has). More precisely: for each rectangle $x$, either they saw $x$ as horizontal, or they saw $x$ as vertical. If the subjects merely say that they saw eight rectangles, some horizontal and some vertical, or that "they can see all or almost all the 8 to 12 items in the presented arrays" (sect. 9, para. 11), this is insufficient.

According to Landman et al., selected stimulus information is transferred from the transient iconic memory to the more durable working memory. Working memory therefore contains less information about the stimulus than iconic memory. If that is all that working memory contains, and if working memory governs subjects' reports about what they see (as Block supposes), then subjects would simply say that they saw a circle of rectangles and saw some of them as oriented horizontally/vertically. They would not, then, agree that they saw details, some of which they can't report. So our third point is this: Block must deny that the contents of working memory are simply a subset of the contents of iconic memory, which is to go beyond the results of Landman et al. If Block is right and subjects report (correctly) that they saw each rectangle as oriented horizontally or vertically, then the contents of working memory should include, not just certain information about the stimulus transferred from iconic memory, but also the meta-information that some information was not transferred. We are not saying that this proposal about the contents of working memory is wrong, but only that the Landman experiment does not address it.

\section{NOTE}

1. The question of the exact relationship between visible and informational persistence remains open. Loftus and Irwin (1998) argue that the many measures of visible and informational persistence pick out the same underlying process. Nevertheless, the distinction is still useful and our discussion does not rely on the assumption that it marks a real difference.

\section{Experience and agency: Slipping the mesh}

\author{
doi: 10.1017/S0140525X07002828
}

\section{Andy Clark and Julian Kiverstein \\ School of Philosophy, Psychology and Language Sciences, George Square, Edinburgh EH8 9JX, Scotland, United Kingdom. \\ andy.clark@ed.ac.uk s9903600@sms.ed.ac.uk \\ http://www.philosophy.ed.ac.uk/staff/clark.html}

Abstract: Can we really make sense of the idea (implied by Block's treatment) that there can be isolated islets of experience that are not even potentially available as fodder for a creature's conscious choices and decisions? The links between experience and the availability of information to guide conscious choice and inform reasoned action may be deeper than the considerations concerning (mere) reportability suggest.

In this elegant and tightly argued target article, Ned Block seeks to persuade us that phenomenal consciousness routinely "overflows" cognitive accessibility. By this he means that we can (and do) have experiences even in cases where we lack the kind of access that would yield some form of report that such and such an experience had occurred. The case Block makes for such an apparently hard-to-support judgment rests on a "mesh" between psychological results and work in neuroscience. The psychological data seem to show that subjects can see much more than working memory enables them to report. Thus, in the Landman et al. (2003) experiments, for instance, subjects show a capacity to identify the orientation of only four rectangles from a group of eight. Yet they typically report having seen the specific orientation of all eight rectangles. Working memory here seems to set a limit on the number of items available for conceptualization and hence report.

Work in neuroscience then suggests that unattended representations, forming parts of strong-but-still-losing clusters of activation in the back of the head, can be almost as strong as the clusters that win, are attended, and hence get to trigger the kinds of frontal activity involved in general broadcasting (broadcasting to the "global workspace"). But whereas Dehaene et al. (2006) treat the contents of such close-seconds as preconscious, because even in principle (given their de facto isolation from winning frontal coalitions) they are unreportable, Block urges us to treat them as phenomenally conscious, arguing that "the claim that they are not conscious on the sole ground of unreportability simply assumes metaphysical correlationism" (sect. 14, para. 9; italics in original). That is to say, it simply assumes what Block seeks to question - that is, that the kind of functional poise that grounds actual or potential report is part of what constitutes phenomenology. Contrary to this way of thinking, Block argues that by treating the just-losing coalitions as supporting phenomenally conscious (but in principle unreportable) experiences, we explain the psychological results in a way that meshes with the neuroscience.

The argument from mesh (which is a form of inference to the best explanation) thus takes as its starting point the assertion that the only grounds we have for treating the just-losing back-of-thehead coalitions as non-conscious is the unreportability of the putative experiences. But this strikes us as false, or at least premature. For underlying the appeal to reportability is, we suspect, a deeper and perhaps more compelling access-oriented concern. It is the concern that any putative conscious experience should be the experience of an agent. The thought here is that we cannot make sense of the image of free-floating experiences, of little isolated islets of experience that are not even potentially available as fodder for a creatures rational choices and considered actions. Evans (1982) rather famously rejects the very idea of such informationally isolated islands of experience. According to Evans, an informational state may underpin a conscious experience only if it (the informational state) is in some sense input to a reasoning subject. To count as a conscious experience an informational state must: 